\title{
Quantitative Analysis of Pain during Mammography Using Electrical Stimulation
}

\author{
Mieko Uchiyama, Yongbum Lee, Utako Shimizu, Rika Saitoh \\ Graduate School of Health Sciences, Niigata University, Niigata, Japan \\ Email: Uchiyama@clg.niigata-u.ac.jp
}

Received 21 August 2015; accepted 18 September 2015; published 21 September 2015

Copyright (C) 2015 by authors and Scientific Research Publishing Inc.

This work is licensed under the Creative Commons Attribution International License (CC BY). http://creativecommons.org/licenses/by/4.0/

c) (i) Open Access

\begin{abstract}
In order to quantitatively measure the degree of pain during mammography, we used a visual analog scale (VAS) and a device for quantitative analysis of perception and pain and performed regression analysis of the relationship between pain degree and VAS score. Twenty-four subjects underwent a typical mammogram and we found no correlation between pain degree using the device compared with VAS scoring. It is presumed that the system enables quantitative measurement and comparison more accurately than subjective markers such as VAS in quantitation of a pain produced in an examination with clearly defined physical size (such as mammography).
\end{abstract}

\section{Keywords}

Mammography, Pain Degree, Electrical Stimulation, Visual Analog Scale

\section{Introduction}

Mammography is widely performed as a standard breast cancer screening method in Japan and Western countries. In mammography, after the papillary glands are adequately stretched, the breast is compressed with a radiolucent paddle for fixation and a reduction in the breast thickness. In examinees undergoing mammography, not only breast compression but also fixation with the neck being rotated or the shoulder being flexed is necessary. These imaging techniques reduce X-ray exposure and ensure the image quality necessary for diagnosis [1].

Mammography screening, which is considered to be of the greatest benefit for early detection of breast cancer, is widely performed as a standardized procedure [2] [3]. Mammography is a radiological test during which the breast is compressed by parallel plates to make breast tissue as even and as thin as possible. Along with compression of the breast, positioning during mammography such as twisting the neck and keeping the arms up is burdensome. It is well known that mammography screening is physically hard on a woman. Many women feel the physical burden during mammography as pain [4]-[8]. 
The International Association for the Study of Pain (IASP) defined a pain as "an unpleasant sensory and emotional experience associated with actual or potential tissue damage, or described in terms of such damage” [9]. Therefore, when a pain is measured in humans, multiple areas including emotional and cognitive/evaluative aspects need to be taken into account comprehensively, in addition to the sensory aspects such as the intensity and location of a pain identified by the subject.

As for the physical and psychological burden experienced by the examinees at the time of mammography positioning, we have previously demonstrated empirically the relationship between muscle activity and pain based on the imaging method and the process of imaging [10]-[12]. Based on these results, we reported that patients experience more than moderate pain from the start of positioning through the entire course of breast compression and immobilization and that pain also occurs at sites other than the breast.

In the past, researchers have used subjective methods to assess pain in subjects undergoing mammography. Sharp et al. [13] used the visual analog scale (VAS) [14] [15] and Hafslund et al. [7] have used the McGill Pain Questionnaire [16]. Although subjective assessment of pain is widely used, it is regarded as inaccurate because it lacks objectivity and reproducibility. This has led to the invention of a method to quantitatively assess pain by applying electrical stimulation and comparing the pain sensation with the sensation of the stimulus [17]. They were those used to measure the pain during mammography. In this study, we quantitatively measured the degree of pain during mammography using this method.

\section{Methods}

The study included 24 healthy women. Their mean age was $24.0 \pm 6.4$ years, mean height was $159.5 \pm 6.6 \mathrm{~cm}$, and mean weight was $52.2 \pm 5.6 \mathrm{~kg}$. Of the 24 subjects, 2 had previously undergone mammography, and 22 had never had mammograms.

The subjects were given explanation on the study objectives, methods, and safety, and their informed consent was obtained. This study was conducted with the approval of the Research Ethics Committee of Niigata University School of Medicine (Approval Number 1946).

For this study, mammography was carried out only up to the completion of positioning, including compression of the breast, and the tissue was not x-rayed. Only positioning for the mediolateral oblique view of the right breast was performed. After the sham mammogram, a device for quantitative analysis of perception and pain (Pain Vision ${ }^{\mathrm{TM}}$ PS-2100; NIPRO Co. Ltd., Japan: Pain Vision ${ }^{\mathrm{TM}}$ ) was used to measure the degree of pain from mammography; at the same time, the subject assessments on the VAS were recorded. The criterion for judging pain measured was defined as maximum pain experienced upon compression of the breast during mammography. Typical thing in subjective pain measurement is VAS. This study is to compare the measured values of the quantitative pain and determination of subjective pain.

The sensory and pain analyzer Pain Vision ${ }^{\mathrm{TM}}$ emits a stimulatory electric current from an electrode attached to the skin; this pulsed electric current (50 - $3000 \mathrm{~Hz} ; 0$ - $150 \mu \mathrm{Ams}$; pulse width, $0.03 \mathrm{~mA}$ ) consists of not only single waves, but differential waves with a sharp tip. With electric stimulation, the pain threshold is measurable with the analyzer. The wave frequency emitted from the system stimulates $\mathrm{A} \beta$ fibers and some $\mathrm{A} \delta$ fibers of sensory nerves. A single wave with a sharp edge enables efficient stimulation of $\mathrm{A} \beta$ fibers and provides the subject with heteresthesia without pain [18].

The principle of measurement is based on the inability of humans to separately evaluate pain at more than one site simultaneously. At measurement, the minimum sensed electric current is measured three times, and the average electric current is defined as the minimum sensed threshold. Next, an electric current for electric stimulation is gradually increased and the subject is asked to push a button to indicate when he/she felt comparable pain intensity. That electric current level is defined as the pain-equivalent electric current. The reason why the minimum sensible electric current threshold is set up is to eliminate any inter-individual difference in recognizing relative positioning of electrodes and subcutaneous nerves and sensing it in the brain. With this system, "pain intensity" is calculated based on the pain equivalent electric current obtained in this manner and the minimum sensible electric current threshold according to the following formula (1).

$$
\text { Pain degree }=\frac{\text { (a pain equivalent electric current }- \text { a minimum sensible threshold })}{\text { a minimum threshold }}
$$

For measurement, a disposable distal electrode (EL-BAND, NIPRO) is attached on the medial side of the arm, 
$1 \mathrm{~cm}$ from the midpoint between the center of the bend of the elbow and the center of the wrist on the flexor side of the forearm (Figure 1). Pain degree of the measurement results are displayed in the bottom left of the screen (Figure 2).

The VAS is an assessment method of pain due to subjective factors. The subjects were asked to point to a position along a 100-mm line with 1-mm increments, its left end (0 points) representing "no pain" and right end (100 points) representing "worst pain imaginable," to indicate the level of pain they are experiencing as a score. Figure 3 shows a diagram of the VAS. The participants were asked to point with their finger to the level of pain they were feeling during whole mammography.

Regression analysis was performed to define the relationship between degree of pain and VAS score. Statistical analyses were performed using SPSS 19J for Windows and the significance level was set at less than 0.05 .

\section{Results}

For the 24 subjects studied, the mean VAS score (and standard deviation) during mammography was $51.0 \pm$ 28.0, and the mean degree of pain value (and standard deviation) was $388.7 \pm 355.0$. A guideline for moderate to severe pain on the VAS is a score of $\geq 40$, and the results of our study showed that the subjects had experienced at least moderate pain during mammography. Degree of pain can be said, in theory, to be "no pain" if the score is between 0 and 100, and a "perceivable quantity of stimulus" if the score is $>100$, suggesting that the subjects in our study experienced intense pain during mammography. However, there was a wide variation in values on the degree of pain measurement.

The relationship between VAS and degree of pain was $y=68.10 x+23.766, r^{2}=0.289(p<0.01)$, which showed no correlation (Figure 4). The values vary considerably, including cases where the VAS score was $>60$ although the degree of pain was $<100$, and cases where the degree of pain $>200$ although the VAS score was $<10$.

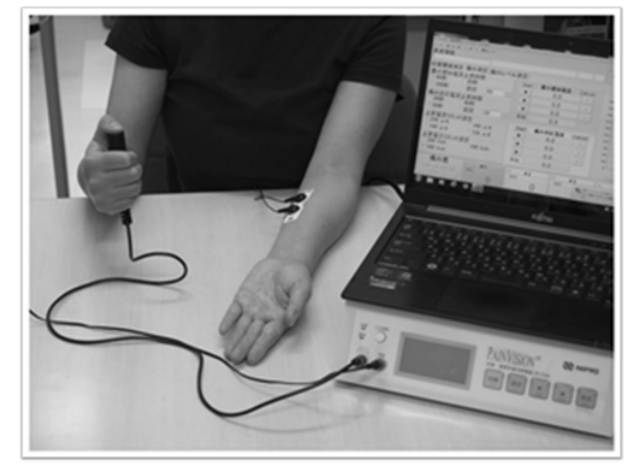

Figure 1. Measurements of pain degree that depends on the pain vision. For measurement, a disposable distal electrode (EL-BAND, NIPRO) is attached on the medial side of the arm, $1 \mathrm{~cm}$ from the midpoint between the center of the bend of the elbow and the center of the wrist on the flexor side of the forearm. An electric current for electric stimulation is gradually increased and the subject is asked to push a button.

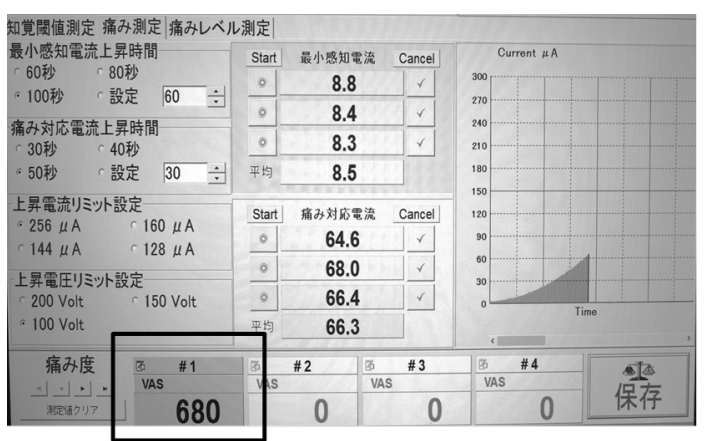

Figure 2. Computer display screen at the time of pain degree measurement. Displayed of minimum sensing current value (current perception threshold), pain corresponding current value and pain degree in Japanese version. 

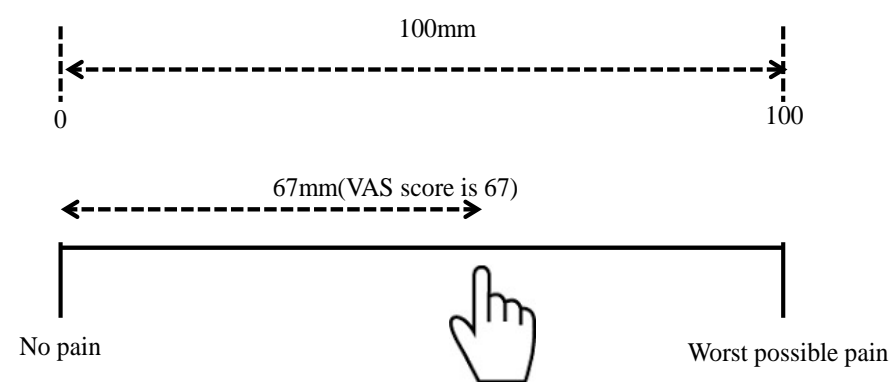

Figure 3. Visual analog scale for subjective pain assessment.

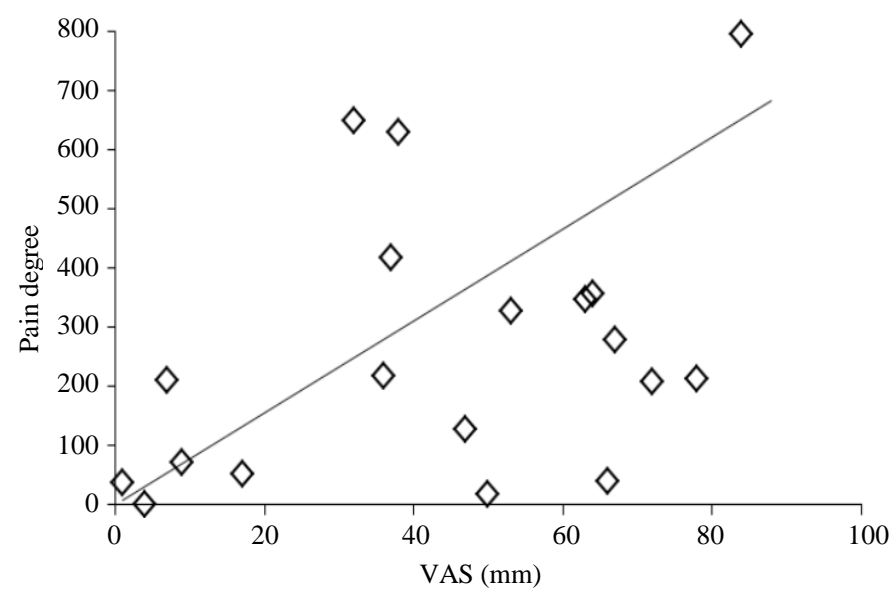

Figure 4. The correlation coefficient of the degree of pain during mammography assessed by degree of pain evaluation and VAS score.

\section{Discussion}

Whereas the VAS score is assessed within a range of 0 to 100, the degree of pain (without such range) can assess pain of a similar degree, as induced pain is converted to the pain one is actually experiencing. In situations where pain is largely affected by mental factors, VAS scores judge pain that has been subjectively overrated; however, the degree of pain score is considered to be less affected by psychological factors, because it can assess the degree of pain from a physical stimulus actually applied by the device.

A study on the effects of pain treatment using Pain Vision ${ }^{\mathrm{TM}}$ versus VAS scoring showed a strong correlation between the VAS score and degree of pain [19]. In our study, no significant correlation between the VAS score and degree of pain was found. This may have been due to psychological factors related to mammography and the temporal error caused by the delay between mammography and measurement of pain. However, one subject showed a moderate pain score of 40 on the VAS, whereas her pain degree score was high (approximately 700). Although the values vary between individuals, quantified measurements of pain or strain may allow for examination of various factors of pain during mammography. Further investigation of a greater number of subjects is needed.

This suggests that they influenced not only the pain and burden associated with breast compression directly involved in mammography but also a physical burden on the whole body and a psychological burden continuously present from the time before the screening, and were related to the results we had previously verified [20]-[23].

The results of this study suggested a few candidate situations for using the Pain Vision ${ }^{\mathrm{TM}}$ system. The first situation is in combination with a visual analog scale (VAS). As shown in the definition of pain by IASP, a pain is not measurable based solely on physical size. Since there is a difference in subjective sense of a pain, the threshold of a pain changes.

However, it is presumed that the system enables quantitative measurement and comparison more accurately than subjective markers such as VAS in quantitation of a pain produced in an examination with clearly defined 
physical size (such as mammography). It is thought that a combination with VAS is useful for elucidation of not only pain intensity but also the nature of a pain.

The other situation is evaluation of the benefit of care when intervention is considered for reduction of a pain. It is expected that the system will be used as a common tool in a large-scale survey for measurement of the effect because of the portability and potential availability of the equipment.

\section{Conclusion}

We quantitatively measured the degree of pain during mammography using Pain Vision ${ }^{\mathrm{TM}}$ and compared it to VAS scores. Twenty-four subjects underwent mammography and we found no correlation between degree of pain and VAS score. In the future, we believe that we should be verified in a larger number of samples.

\section{Conflict of Interest}

The authors declare that they have no conflict of interest.

\section{Acknowledgements}

This work was supported by JSPS KAKENHI Grant Numbers 26463372.

\section{References}

[1] Henrich, R.E., Baett, L., Botsco, M.A., Deibei, D., Feig, S. and Gray, J. (1999) Mammography Quality Control Manual: Patient Positioning and Compression. American College of Radiology, 30-75.

[2] Drukker, B.H. (1997) Breast Disease: A Primer on Diagnosis and Management. International Journal of Fertility and Women's Medicine, 42, 278-287.

[3] Kerlikowske, K., Grady, D., Rubin, S.M., Sandrock, C. and Ernster, V.L. (1995) Efficacy of Screening Mammography. A Meta-Analysis. Journal of the American Medical Association, 273, 149-154. http://dx.doi.org/10.1001/jama.1995.03520260071035

[4] Aro, A.R., Absetz-Ylöstalo, P., Eerola, T., Pamilo, M. and Lönnqvist, J. (1996) Pain and Discomfort during Mammography. European Journal of Cancer, 32A, 1674-1679. http://dx.doi.org/10.1016/0959-8049(96)00140-2

[5] Kornguth, P.J., Keefe, F.J. and Conaway, M.R. (1996) Pain during Mammography: Characteristics and Relationship to Demographic and Medical Variables. Pain, 66, 187-194. http://dx.doi.org/10.1016/0304-3959(96)03057-6

[6] Asghari, A. and Nicholas, M.K. (2004) Pain during Mammography: The Role of Coping Strategies. Pain, 108, $170-179$. http://dx.doi.org/10.1016/i.pain.2003.12.022

[7] Hafslund, B. (2000) Mammography and the Experience of Pain and Anxiety. Radiography, 6, 269-272. http://dx.doi.org/10.1053/radi.2000.0281

[8] Sapir, R., Patlas, M., Strano, S.D., Hadas-Halpern, I. and Cherny, N.I. (2003) Does Mammography Hurt? Journal of Pain and Symptom Management, 25, 53-63. http://dx.doi.org/10.1016/S0885-3924(02)00598-5

[9] Merskey, H and Bogduk, N. (1994) Classification of Chronic Pain: Descriptions of Chronic Pain Syndromes and Definitions of Pain Terms. IASP Press, 1-28.

[10] Uchiyama, M., Lee, Y., Kazama, K., Minagawa, Y., Tsai, D.Y., Sadakata, M. and Sayama, M. (2011) A Preliminary Study on Measurement of Muscle Activity during Mammography Positioning. Japanese Journal of Radiological Technology, 67, 679-682. http://dx.doi.org/10.6009/jjrt.67.679

[11] Uchiyama, M., Lee, Y., Sadakata, M., Sayama, M. and Tsai, D.Y. (2012) Measurement of Muscle Activities for Evaluating Physical Burden and Pain during Mammography Positioning. The Tohoku Journal of Experimental Medicine, 228, 53-58. http://dx.doi.org/10.1620/tjem.228.53

[12] Uchiyama, M., Lee, Y., Kazama, K., Minagawa, Y., Tsai, D.Y., Sadakata, M. and Sayama, M. (2012) Quantitative Assessment of Muscle Activity in Mammography Positioning. 20th IMEKO World Congress, Busan, 9-14 September 2012, 9-14.

[13] Sharp, P.C., Michielutte, R., Freimanis, R., Cunningham, L., Spangler, J. and Burnette, V. (2003) Reported Pain Following Mammography Screening. Archives of Internal Medicine, 163, 833-836. http://dx.doi.org/10.1001/archinte.163.7.833

[14] Keel, K.D. (1948) The Pain Chart. Lancet, 2, 6-8. http://dx.doi.org/10.1016/S0140-6736(48)91787-5

[15] Turk, D.C. and Melzack, R. (2001) Handbook of Pain Assessment. 2nd Edition, The Guilford Press, New York. 
[16] Merzack, R. (1975) The McGill Pain Questionnaire: Major Properties and Scoring Methods. Pain, 1, 277-299. http://dx.doi.org/10.1016/0304-3959(75)90044-5

[17] Dinash, G., Daniel, K., Len, G. and John, G. (2001) Examining the Validity of Pressure Ulcer Risk Assessment Scales: Developing and Using Illustrated Patient Simulations to Collect The Data. Journal of Clinical Nursing, 10, 697-706. http://dx.doi.org/10.1046/j.1365-2702.2001.00525.x

[18] Shimazu H., Seino, S.-I., Kato, S., Kobayashi, H. and Akimoto, M. (2005) Development of a Quantitative Measurement Method for the Magnitude of Pain Using Painless Electrical Stimulation and Its Evaluation Using Experimental Pain. Transactions of Japanese Society for Medical and Biological Engineering, 43, 117-123.

[19] Hasegawa, J., Sugiyama, D., kumasaka, M., Hishinuma, M., Matsuo, K., Ide, S., Tanaka, S. and Kitoh, T. (2008) Evaluation of Pain Treatment by Pain Vision ${ }^{\text {TM }}$. Journal of Japan Society of Pain Clinicians, 15, 144-149.

[20] Uchiyama, M., Lee, Y., Sadakata, M., Tsai, D.Y. and Sayama, M. (2013) Effects of Mammography Positioning on the Autonomic Nervous Function. Health, 5, 1335-1341. http://dx.doi.org/10.4236/health.2013.58181

[21] Lee, Y. and Uchiyama, M. (2014) Evaluation of Physical and Psychological Burden of Subjects in Mammography. IWDM 2014, LNCS, 8539, 508-513. http://dx.doi.org/10.1007/978-3-319-07887-8_71

[22] Uchiyama, M. (2014) The Experience of Mammography Based on the Memoirs of Examinees. Health, 6, $1310-1314$. http://dx.doi.org/10.4236/health.2014.611160

[23] Lee, Y. and Uchiyama, M. (2015) The Effect of Humorous Stimuli on Alleviating Pain during Mammography: A Preliminary Study. Health, 7, 659-664. http://dx.doi.org/10.4236/health.2015.76078 